\title{
Fifteen per cent of people treated for mental health disorders are homeless
}

Folsom DP, Hawthorne W, Lindamer L, et al. Prevalence and risk factors for homelessness and utilization of mental health services among 10,340 patients with serious mental illness in a large public mental health system. Am J Psychiatry 2005;162:370-6.

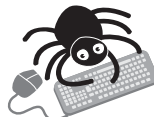

This article

contains extra text on the EBMH website

What is the prevalence of homelessness in people treated for mental health disorders?

\section{METHODS}

$\square$

Design: Cross sectional study.

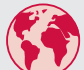

Setting: Adult Mental Health Services database in San Diego County, USA, for the fiscal year 1999-2000.

으 Population: 10340 adults with a diagnosis of schizophrenia bipolar disorder, or major depression, who received treatment at least once during the year, and had data available for ethnicity, living situation, and Global Assessment of Functioning (GAF) score. People in jail or locked psychiatric facilities were excluded.

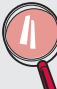

Assessment: People were recorded as homeless if they were living on the street, in a homeless shelter, in a nightly hotel, or a car at the time of treatment in the San Diego County Adult Menta Health Service.

国耍

Outcomes: Homelessness.

\section{MAIN RESULTS}

The overall prevalence of homelessness in people treated for mental health disorders was $15 \%$. Homelessness was most prevalent in people with schizophrenia $(20 \%)$, followed by bipolar disorder $(17 \%)$ and depression (9\%)

\section{CONCLUSIONS}

Homelessness is a problem in people treated for mental health disorders. Research is needed into interventions to reduce homelessness in this population.

\section{NOTES}

The data used in this study were obtained retrospectively from database analysis and were not independently verified. For correspondence: Dr Folsom, Psychiatry Service $(116 A-1)$ VA San Diego Healthcare System, 3350 La Jolla Village Dr., San Diego, CA 92161, USA; dfolsom@ucsd.edu

Sources of funding: National Institute of Mental Health (NIMH) grants, Department of Veterans Affairs Veterans Integrated Service Network 22 Mental Illness Research, Education and Clinical Center.

\section{Commentary}

A n estimated 2.3-3.5 million Americans experience homelessness each year, and mental health problems, which are present in about one third of these individuals, significantly increase the risk of long term homelessness. ' As a result, clinicians working in public mental health systems frequently provide care for people who are homeless. Folsom et al studied a large group of non-institutionalised patients with schizophrenia, bipolar disorder, or major depression who had contact with the public mental health system in San Diego in 19992000. Fifteen per cent of these individuals were homeless at some point over a one year period. In comparison, a previous study of people treated in Philadelphia's public mental health system in the early 1990s found that only $7-10 \%$ of those with schizophrenia or affective psychoses were admitted to a homeless shelter over a three year period. ${ }^{2}$ These contrasting findings raise the worrisome possibility that over the last decade, homelessness may have become much more common among Americans suffering from mental illness.

Not surprisingly, this study found that homelessness was significantly more common among people who were male or African-American, had schizophrenia or bipolar disorder, had a substance use disorder, or had a lower level of functioning. These characteristics were previously known to be risk factors for homelessness. Interestingly, people with mental health problems covered by Medicaid were about half as likely to be homeless than those without Medicaid. This association likely reflects the many barriers to obtaining entitlements that homeless people experience, although the possibility that Medicaid benefits confer some degree of protection against homelessness cannot be ruled out.

This study's use of a large and comprehensive dataset is a notable strength. Although its findings have few direct implications for clinical practice, the study serves as a stark reminder to providers in public mental health systems that their clients' need for stable housing may be equal to or perhaps even greater than their need for treatment of their mental illness.

Stephen W Hwang, MD, MPH Centre for Research on Inner City Health, St Michael's Hospital; Department of Medicine, University of Toronto, Toronto, Canada

1 Burt M, Aron LY, Lee E, et al. Helping America's Homeless: Emergency shelter or affordable housing? Washington, DC: The Urban Institute Press, 2001.

2 Culhane DP, Averyt JM, Hadley TR. The rate of public shelter admission among Medicaid-reimbursed users of behavioural health services. Psychiatr Serv 1997;48:390-2

Please see http://www.ebmentalhealth.com/supplemental for an additional commentary by Daniel W Bradford, Mimi M Kim, and Marian I Butterfield. 